\title{
METHOD OF FINDING AN OPTIMAL SOLUTION TO THE PROBLEM OF INTERVAL ASSIGNMENT
}

\section{G. RAMESH \& G. GAJENDRAN}

Department of Mathematics, SRM Institute of Science and Technology, Kattankulathur, Tamil Nadu, India

\begin{abstract}
Models of transport and assignment play a major role in the Managing the logistics and supply chain to cut costs and Space, Service better. A general problem of assignment includes $N$ tasks that must be delegated to $N$ workers where each worker has the ability to perform all tasks. The goal is to assign a proper worker to each task so as to minimize total resource spending to complete all tasks. The purpose of this paper is to assess the question of an assignment under uncertainty (Interval form) and also transform the problem of assignment intervals into Hungarian method. At the end, a numerical example is given and to find an optimal solution for problem of distribution of intervals.

KEYWORDS: Interval Numbers, Generalized Interval Arithmetic, Interval Linear Programming, Ranking
\end{abstract}

Received: Jun 10, 2020; Accepted: Jun 30, 2020; Published: Aug 04, 2020; Paper Id.: IJMPERDJUN2020614

\section{INTRODUCTION}

In real life we face the question of allocating multiple staff / workers to different occupations. Not everybody is equally capable of performing a given task. Different people have different abilities to perform the same function and these different abilities are reflected in terms of the cost / profit / time involved in the performance of a given job. Therefore, we have to consider whether to assign different workers to different positions in order to reduce the expense of doing such work. The assigned may be employers for who work assignments are needed. Assignment of people to work is a common issue of assignment. The delegated need not be individuals, however. They may even be vehicle or plant unit, or even time slots to delegate tasks. There are thus primarily four methods in the literature, namely Enumeration method, Simplex method, Transportation problem and Hungarian method. Hungarian method is one of the best possible methods to solve an assignment problem. Costs are not always in a smooth form in the realistic problems; sometimes those parameters are unclear which are represented by intervals. Therefore we need the assistance of interval analysis to handle this form of data. In this paper a general issue of linear assignment intervals is taken into account, on the premise that one person will accomplish one job at a time. Use of this proposed method we solved one example problem. Corresponding results are determined and were recorded here. "The best individual for the task" is an apt definition of the model of assignment. A significant issue which was raised immediately after the transport question, Is the problem with the task.

\section{The Study of Literature}

Several methods exist in the literature to solve these kinds of problems. D. Konig, J. Egervary and thus is known as the Hungarian assignment models method [1]. They suggested G.Ramesh and Ganesan and Deepa[2, 4] Current programming strategy to solve generalized assignment problem Hungarian Interval System. Dr. S. Amutha, S. 
Lakshmi, S. Narmatha[5] developed by method to solve interval extension in Assignment Cloud problem, Michael J. Moore, Raman E Kearfott. R. Baker [6], Introduction to an study of intervals. In the problem of random assignment Aldous[7] studied asymptotes. Pardalos and Pitsoulis[8] established some work on problems with nonlinear assignments. K. Ganesan and P. Veeramani studied by using a new method of comparing generalized interval numbers and a new set of generalized interval arithmetic [9, 10], Veeramani develops a simplex like algorithm to solve linear interval programming problems without converting them to classical linear programming issues. Mraz[11] determines the exact upper and lower limits of optimum values for linear programming problems, the coefficients of which differ at a given interval. [12] D Priya and G Ramesh developed by the Hungarian Assignment Problem Method, With Generalized Arithmetic Intervals and Applications;

The rest of the paper is structured according to: Highlighted in the next section on interval arithmetic. Examples of the proposed Hungarian interval approach are described in section 3. Problems are solved in section 4 and findings are evaluated. Conclusions are eventually drawn in section 5.

\section{Preliminaries}

The aim of this section is to present some remarks, ideas and findings that are of use in our further consideration.

\section{Interval Numbers}

Let $\tilde{a}=\left[a_{1}, a_{2}\right]=\left\{x: a_{1} \leq x \leq a_{2}, x \in R\right\}$. If $\tilde{a}=a_{1}=a_{2}=a$, then $\tilde{a}=[a, a]=a$ is a real number (or a degenerate interval). Let IR $=\left\{\tilde{a}=\left[a_{1}, a_{2}\right]: a_{1} \leq a_{2}\right.$ and $\left.a_{1}, a_{2} \in R\right\}$ be the set of all proper intervals and $\overline{I R}=\left\{\tilde{a}=\left[a_{1}, a_{2}\right]: a_{1}>a_{2}\right.$ and $\left.a_{1}, a_{2} \in R\right\}$ be the set of all improper intervals on the real line R. We shall use the terms interval and interval number interchangeably.

The mid-point and width (or half-width) of an interval number $\tilde{a}=\left[a_{1}, a_{2}\right]$ are defined as $m(\tilde{a})=\left(\frac{a_{1}+a_{2}}{2}\right)$ and $\mathrm{w}(\tilde{\mathrm{a}})=\left(\frac{\mathrm{a}_{2}-\mathrm{a}_{1}}{2}\right)$. The interval number $\tilde{\mathrm{a}}$ can also be expressed in terms of its midpoint and width as $\tilde{\mathrm{a}}=\left[\mathrm{a}_{1}, \mathrm{a}_{2}\right]=\langle\mathrm{m}(\tilde{\mathrm{a}}), \mathrm{w}(\tilde{\mathrm{a}})\rangle$.

\section{Ranking of Interval Numbers}

Sengupta and Pal[13] suggested a easy and powerful index to compare any two intervals on IR through the satisfaction of decision-makers.

Definition: Let $\preceq$ be an extended order relation between the interval numbers $\tilde{a}=\left[a_{1}, a_{2}\right], \tilde{b}=\left[b_{1}, b_{2}\right]$ in IR, then for $m(\tilde{a})<m(\tilde{b})$, we construct a premise $\left(\tilde{a}^{\circ} \tilde{b}\right)$ which implies that $\tilde{a}$ is inferior to $\tilde{b}$ (or $\tilde{b}$ is superior to $\tilde{a}$ ).

An acceptability function $\mathrm{A}_{\preceq}: \operatorname{IR} \times \mathrm{IR} \rightarrow[0, \infty)$ is defined as:

$$
\mathrm{A}_{\preceq}(\tilde{\mathrm{a}}, \tilde{\mathrm{b}})=\mathrm{A}(\tilde{\mathrm{a}} \preceq \tilde{\mathrm{b}})=\frac{\mathrm{m}(\tilde{\mathrm{b}})-\mathrm{m}(\tilde{\mathrm{a}})}{\mathrm{w}(\tilde{\mathrm{b}})+\mathrm{w}(\tilde{\mathrm{a}})}, \text { where } \mathrm{w}(\tilde{\mathrm{b}})+\mathrm{w}(\tilde{\mathrm{a}}) \neq 0 .
$$

$\mathrm{A}_{\prec} \quad$ may be interpreted as the grade of acceptability of the first interval number to be inferior to the second interval number. For any two interval numbers $\tilde{a}$ and $\tilde{b}$ in $\operatorname{IR}$ either $\mathrm{A}(\tilde{\mathrm{a}} \preceq \tilde{\mathrm{b}}) \geq 0$ (or) $\mathrm{A}(\tilde{\mathrm{b}} \succeq \tilde{\mathrm{a}}) \succeq 0$ (or) $\mathrm{A}(\tilde{\mathrm{a}} \preceq \tilde{\mathrm{b}})=0$ (or) $\mathrm{A}(\tilde{\mathrm{b}} \succeq \tilde{\mathrm{a}})=0$ (or) $\mathrm{A}(\tilde{\mathrm{a}} \preceq \tilde{\mathrm{b}})+\mathrm{A}(\tilde{\mathrm{b}} \preceq \tilde{\mathrm{a}})=0$. 
If $\mathrm{A}(\tilde{\mathrm{a}} \preceq \tilde{\mathrm{b}})=0$ and $\mathrm{A}(\tilde{\mathrm{b}} \preceq \tilde{\mathrm{a}})=0$, then we say that the interval Numbers $\tilde{\mathrm{a}}$ and $\tilde{\mathrm{b}}$ are equivalent (non-inferior to each other) and we denote it by $\tilde{a} \approx \tilde{b}$. Also if $A(\tilde{a} \preceq \tilde{b}) \geq 0$, then $\tilde{a} \preceq \tilde{b}$ and if $A(\tilde{b} \preceq \tilde{a}) \geq 0$, then $\tilde{b} \preceq \tilde{a}$.

\section{A New Interval Arithmetic}

Ming Ma et al.[14] suggested a new fuzzy arithmetic focused on the index of locations and the index function of fuzziness. For the ordinary arithmetic the position index number is taken, while in the lattice $\mathrm{L}$ the fuzziness index functions are assumed to obey the lattice law which is the least upper bound and the greatest lower bound. That is for $a, b \in \mathrm{L}$ we define $a \vee b=\max \{a, b\}$ and $a \wedge b=\min \{a, b\}$.

For any two intervals $\tilde{a}=\left[a_{1}, a_{2}\right], \tilde{b}=\left[b_{1}, b_{2}\right] \in \mathbb{I R}$ and for $* \in\{+,-, \cdot, \div\}$, the arithmetic operations on $\tilde{a}$ and $\tilde{b}$ are defined as:

$$
\tilde{a} * \tilde{b}=\left[a_{1}, a_{2}\right] *\left[b_{1}, b_{2}\right]=\langle m(\tilde{a}), w(\tilde{a})\rangle *\langle m(\tilde{b}), w(\tilde{b})\rangle=\langle m(\tilde{a}) * m(\tilde{b}), \max \{w(\tilde{a}), w(\tilde{b})\}\rangle .
$$

In particular

$$
\begin{aligned}
& \text { (i). Addition : } \tilde{a}+\tilde{b}=\langle\mathrm{m}(\tilde{\mathrm{a}}), \mathrm{w}(\tilde{\mathrm{a}})\rangle+\langle\mathrm{m}(\tilde{\mathrm{b}}), \mathrm{w}(\tilde{\mathrm{b}})\rangle \quad=\langle\mathrm{m}(\tilde{\mathrm{a}})+\mathrm{m}(\tilde{\mathrm{b}}), \max \{\mathrm{w}(\tilde{\mathrm{a}}), \mathrm{w}(\tilde{\mathrm{b}})\}\rangle \text {. } \\
& \text { (ii). Subtraction : } \tilde{a}-\tilde{b}=\langle\mathrm{m}(\tilde{\mathrm{a}}), \mathrm{w}(\tilde{\mathrm{a}})\rangle-\langle\mathrm{m}(\tilde{\mathrm{b}}), \mathrm{w}(\tilde{\mathrm{b}})\rangle=\langle\mathrm{m}(\tilde{\mathrm{a}})-\mathrm{m}(\tilde{\mathrm{b}}), \max \{\mathrm{w}(\tilde{\mathrm{a}}), \mathrm{w}(\tilde{\mathrm{b}})\}\rangle \text {. } \\
& \text { (iii). Multiplication : } \tilde{a} \times \tilde{b}=\langle m(\tilde{a}), w(\tilde{a})\rangle \times\langle m(\tilde{b}), w(\tilde{b})\rangle=\langle m(\tilde{a}) \times m(\tilde{b}), \max \{w(\tilde{a}), w(\tilde{b})\}\rangle \text {. } \\
& \text { (iv). Division : } \tilde{a} \div \tilde{b}=\langle\mathrm{m}(\tilde{\mathrm{a}}), \mathrm{w}(\tilde{\mathrm{a}})\rangle \div\langle\mathrm{m}(\tilde{\mathrm{b}}), \mathrm{w}(\tilde{\mathrm{b}})\rangle \quad=\langle\mathrm{m}(\tilde{\mathrm{a}}) \div \mathrm{m}(\tilde{\mathrm{b}}), \max \{\mathrm{w}(\tilde{\mathrm{a}}), \mathrm{w}(\tilde{\mathrm{b}})\}\rangle, \\
& \text { provided } \mathrm{m}(\tilde{\mathrm{b}}) \neq \tilde{0} \text {. }
\end{aligned}
$$

\section{MAIN RESULTS}

\section{General Interval Assignment Problem}

Let there be $\mathrm{n}$ work, and $\mathrm{n}$ persons with specific credentials. If $\mathrm{i}^{\text {th }}$ individual does $\mathrm{j}^{\text {th }}$ work the $\cos$ is $\mathrm{C}_{\mathrm{ij}}$. Now the question is the job is to be delegated to whom, in order to minimize the cost of completing the work. We can express the problem in mathematical terms as follows:

To minimize $\tilde{Z}(\operatorname{cost})=\sum_{\mathrm{i}=1}^{\mathrm{n}} \sum_{\mathrm{j}=1}^{\mathrm{n}} \tilde{\mathrm{c}}_{\mathrm{ij}} \tilde{\mathrm{x}}_{\mathrm{ij}} ;[\mathrm{i}=1,2, \ldots \mathrm{n} ; \mathrm{j}=1,2, \ldots \mathrm{n}]$

where $\tilde{x}_{\mathrm{ij}}=\left\{\begin{array}{l}1 ; \text { if } \mathrm{i}^{\text {th }} \text { person is assigned } \mathrm{j}^{\text {th }} \text { work } \\ 0 ; \text { if } \mathrm{i}^{\text {th }} \text { person is not assigned the } \mathrm{j}^{\text {th }} \text { work with the restrictions }\end{array}\right.$

- $\quad \sum_{\mathrm{i}=1}^{\mathrm{n}} \tilde{\mathrm{x}}_{\mathrm{ij}}=1 ; \mathrm{j}=1,2, \ldots$..., i.e., $\mathrm{i}^{\text {th }}$ person will do only one work.

- $\quad \sum_{j=1}^{n} \tilde{x}_{i j}=1 ; i=1,2, \ldots$ n., i.e., $j^{\text {th }}$ work will be done only by one person.

\section{Interval Hungarian Method}


Level 1: Locate the mid values in the cost matrix for each interval.

Level 2: Subtract the interval that has the smallest mid-value in each row from all the row entries.

Level 3: Subtract the interval that has the smallest mid-value from those columns that do not have intervals that have zero from all the column entries.

Level 4: Draw lines through correct rows and columns, such that none of the cost matrix is filled in all intervals and the minimum number of these lines is used.

Level 5: Optimality check (I) If the total number of covering lines is equal to the order of the matrix of costs, then optimality is achieved. (ii) If the total number of covering lines is smaller than the matrix order then proceed to step 6.

Level 6: Determine the lowest interval mid value that is not filled by any rows. Subtract this entry from all elements that are uncrossed, and add it to the crossing that contains zero. Then move on to stage 4

\section{Tabular form of the Problem}

In Table 1 below is given the cost matrix of the interval assignment problem:

Table 1: Cost Matrix of the Interval Assignment Problem

\begin{tabular}{|c|c|c|c|c|c|}
\hline Rersons & 1 & 2 & 3 & $\ldots . . . j$ & $\ldots . . n$ \\
\hline 1 & $\tilde{\mathrm{C}}_{11}$ & $\tilde{\mathrm{C}}_{12}$ & $\tilde{\mathrm{C}}_{13}$ & $\ldots . . \tilde{\mathrm{C}}_{1 \mathrm{j}}$ & $\ldots . . \tilde{\mathrm{C}}_{1 \mathrm{n}}$ \\
\hline 2 & $\tilde{\mathrm{C}}_{21}$ & $\tilde{\mathrm{C}}_{22}$ & $\tilde{\mathrm{C}}_{23}$ & $\ldots . . \tilde{\mathrm{C}}_{2 \mathrm{j}}$ & $\ldots . . \tilde{\mathrm{C}}_{2 \mathrm{n}}$ \\
\hline$\dot{.}$ & $\begin{array}{l}\cdot \\
\cdot \\
\tilde{\mathrm{C}}_{\mathrm{i} 1} \\
\end{array}$ & $\begin{array}{c}\cdot \\
\dot{\cdot} \\
\tilde{\mathrm{C}}_{\mathrm{i} 2} \\
\end{array}$ & $\begin{array}{c}\dot{\cdot} \\
\dot{\tilde{\mathrm{C}}_{\mathrm{i} 3}} \\
\end{array}$ & $\begin{array}{c}\cdot \\
\dot{\tilde{C}_{\mathrm{ij}}}\end{array}$ & $\begin{array}{c}\cdot \\
\dot{\mathrm{C}_{\text {in }}} \\
\end{array}$ \\
\hline$\dot{.} \cdot \dot{ }$ & $\begin{array}{c}\cdot \\
\dot{\cdot} \\
\tilde{\mathrm{C}}_{\mathrm{n} 1}\end{array}$ & $\begin{array}{c}\dot{\cdot} \\
\dot{\mathrm{C}_{\mathrm{n} 2}}\end{array}$ & $\begin{array}{c}\cdot \\
\dot{\cdot} \\
\tilde{\mathrm{C}}_{\mathrm{n} 3}\end{array}$ & $\begin{array}{c}\ldots \ldots \cdot \\
\ldots \ldots \\
\ldots \ldots . \\
\ldots \ldots \tilde{\mathrm{C}}_{n j}\end{array}$ & $\begin{array}{c}\ldots \ldots \\
\ldots \ldots \\
\ldots \ldots \\
\ldots \ldots \tilde{\mathrm{C}}_{\mathrm{nn}}\end{array}$ \\
\hline
\end{tabular}

\section{Numerical Results}

We take the problem of linear assignment as an example and solved this problem using traditional Hungarian method. Assign the four jobs to the four machines, thereby reducing the total cost. In the table below[15], the assignment costs for assigning any operator to any machine are given.

Table 2: Convert the Problem of Assignment of Intervals to the Regular Matrix of Costs by Using Range

\begin{tabular}{|c|c|c|c|c|}
\hline & $\mathbf{J}_{\mathbf{1}}$ & $\mathbf{J}_{\mathbf{2}}$ & $\mathbf{J}_{\mathbf{3}}$ & $\mathbf{J}_{\mathbf{4}}$ \\
\hline $\mathrm{A}$ & {$[10,20]$} & {$[10,15]$} & {$[2,15]$} & {$[0,15]$} \\
\hline $\mathrm{B}$ & {$[1,4]$} & {$[3,12]$} & {$[5,23]$} & {$[4,7]$} \\
\hline C & {$[5,15]$} & {$[2,9]$} & {$[3,6]$} & {$[6,8]$} \\
\hline D & {$[2,7]$} & {$[4,15]$} & {$[4,13]$} & {$[2,9]$} \\
\hline
\end{tabular}


Table 3: Form of Mid Point and Width Cost Matrix with Interval Entries

\begin{tabular}{|c|c|c|c|c|}
\hline & $\mathbf{J}_{1}$ & $\mathbf{J}_{2}$ & $\mathbf{J}_{3}$ & $\mathbf{J}_{4}$ \\
\hline $\mathrm{A}$ & $\langle 15,5\rangle$ & $\langle 12.5,2.5\rangle$ & $\langle 8.5,6.5\rangle$ & $\langle 7.5,7.5\rangle$ \\
\hline $\mathrm{B}$ & $\langle 2.5,1.5\rangle$ & $\langle 7.5,4.5\rangle$ & $\langle 14,9\rangle$ & $\langle 5.5,1.5\rangle$ \\
\hline $\mathrm{C}$ & $\langle 10,5\rangle$ & $\langle 5.5,3.5\rangle$ & $\langle 4.5,1.5\rangle$ & $\langle 7,1\rangle$ \\
\hline $\mathrm{D}$ & $\langle 4.5,2.5\rangle$ & $\langle 9.5,5.5\rangle$ & $\langle 8.5,5.5\rangle$ & $\langle 5.5,3.5\rangle$ \\
\hline
\end{tabular}

Table 4: Row Reduction Cost Matrix with Interval Entries

\begin{tabular}{|c|c|c|c|c|}
\hline & $\mathbf{J}_{\mathbf{1}}$ & $\mathbf{J}_{\mathbf{2}}$ & $\mathbf{J}_{\mathbf{3}}$ & $\mathbf{J}_{\mathbf{4}}$ \\
\hline $\mathrm{A}$ & $\langle 7.5,7.5\rangle$ & $\langle 5,7.5\rangle$ & $\langle 1,7.5\rangle$ & $\langle 0,7.5\rangle$ \\
\hline $\mathrm{B}$ & $\langle 0,1.5\rangle$ & $\langle 5,4.5\rangle$ & $\langle 11,9\rangle$ & $\langle 3,1.5\rangle$ \\
\hline $\mathrm{C}$ & $\langle 5.5,5\rangle$ & $\langle 1,3.5\rangle$ & $\langle 0,1.5\rangle$ & $\langle 2.5,1\rangle$ \\
\hline $\mathrm{D}$ & $\langle 0,2.5\rangle$ & $\langle 5,5.5\rangle$ & $\langle 4,5.5\rangle$ & $\langle 1,3.5\rangle$ \\
\hline
\end{tabular}

Table 5: Column Reduction Cost Matrix With Interval Entries

\begin{tabular}{|c|c|c|c|c|}
\hline & $\mathbf{J}_{\mathbf{1}}$ & $\mathbf{J}_{\mathbf{2}}$ & $\mathbf{J}_{\mathbf{3}}$ & $\mathbf{J}_{\mathbf{4}}$ \\
\hline $\mathrm{A}$ & $\langle 7.5,7.5\rangle$ & $\langle 4,7.5\rangle$ & $\langle 1,7.5\rangle$ & $\langle 0,7.5\rangle$ \\
\hline $\mathrm{B}$ & $\langle 0,1.5\rangle$ & $\langle 4,4.5\rangle$ & $\langle 11,9\rangle$ & $\langle 3,1.5\rangle$ \\
\hline $\mathrm{C}$ & $\langle 5.5,5\rangle$ & $\langle 0,3.5\rangle$ & $\langle 0,1.5\rangle$ & $\langle 2.5,1\rangle$ \\
\hline $\mathrm{D}$ & $\langle 0,2.5\rangle$ & $\langle 4,5.5\rangle$ & $\langle 4,5.5\rangle$ & $\langle 1,3.5\rangle$ \\
\hline
\end{tabular}

Table 6: Cost Matrix With Interval Entries

\begin{tabular}{|c|c|c|c|c|}
\hline & $\mathbf{J}_{\mathbf{1}}$ & $\mathbf{J}_{\mathbf{2}}$ & $\mathbf{J}_{\mathbf{3}}$ & $\mathbf{J}_{\mathbf{4}}$ \\
\hline $\mathrm{A}$ & $\langle 7.5,7.5\rangle$ & $\langle 4,7.5\rangle$ & $\langle 1,7.5\rangle$ & $\langle 0,7.5\rangle$ \\
\hline B & $\langle 0,1.5\rangle$ & $\langle 4,4.5\rangle$ & $\langle 11,9\rangle$ & $\langle 3,1.5\rangle$ \\
\hline C & $\langle 5.5,5\rangle$ & $\langle 0,3.5\rangle$ & $\langle 0,1.5\rangle$ & $\langle 2.5,1\rangle$ \\
\hline D & $\langle 0,2.5\rangle$ & $\langle 4,5.5\rangle$ & $\langle 4,5.5\rangle$ & $\langle 1,3.5\rangle$ \\
\hline
\end{tabular}

Table 5: Cost Matrix With Interval Entries

\begin{tabular}{|c|c|c|c|c|}
\hline & $\mathbf{J}_{1}$ & $\mathbf{J}_{2}$ & $\mathbf{J}_{3}$ & $\mathbf{J}_{4}$ \\
\hline A & $\langle 8.5,7.5\rangle$ & $\langle 3,7.5\rangle$ & $\langle 0,7.5\rangle$ & $30,7.52$ \\
\hline $\mathrm{B}$ & $\langle 0,1.5\rangle$ & $\langle 2,7.5\rangle$ & $\langle 9,9\rangle$ & $\langle 2,3.5\rangle$ \\
\hline $\mathrm{C}$ & $\langle 7.5,5\rangle$ & $\langle 0,3.5\rangle$ & $30,1.5\rangle$ & $\langle 3.5,7.5\rangle$ \\
\hline $\mathrm{D}$ & $37,2.55$ & $\langle 2,7.5\rangle$ & $\langle 2,7.5\rangle$ & $\langle 0,3.5\rangle$ \\
\hline
\end{tabular}

Table 6: Optimal Assignment Cost Matrix With Interval Entries

\begin{tabular}{|c|c|c|c|c|}
\hline & $\mathbf{J}_{\mathbf{1}}$ & $\mathbf{J}_{\mathbf{2}}$ & $\mathbf{J}_{\mathbf{3}}$ & $\mathbf{J}_{\mathbf{4}}$ \\
\hline $\mathrm{A}$ & $\langle 15,5\rangle$ & $\langle 12.5,2.5\rangle$ & $\langle 8.5,6.5\rangle$ & $\langle 7.5,7.5\rangle$ \\
\hline $\mathrm{B}$ & $\langle 2.5,1.5\rangle$ & $\langle 7.5,4.5\rangle$ & $\langle 14,9\rangle$ & $\langle 5.5,1.5\rangle$ \\
\hline $\mathrm{C}$ & $\langle 10,5\rangle$ & $\langle 5.5,3.5\rangle$ & $\langle 4.5,1.5\rangle$ & $\langle 7,1\rangle$ \\
\hline $\mathrm{D}$ & $\langle 4.5,2.5\rangle$ & $\langle 9.5,5.5\rangle$ & $\langle 8.5,5.5\rangle$ & $\langle 5.5,3.5\rangle$ \\
\hline
\end{tabular}

The optimal assignment to computers for all workers is as follows:

$$
\begin{aligned}
& \mathbf{A} \rightarrow \mathbf{J}_{1}, \mathbf{B} \rightarrow \mathbf{J}_{3}, \mathbf{C} \rightarrow \mathbf{J}_{2}, \mathbf{D} \rightarrow \mathbf{J}_{\mathbf{4}} \\
& =\langle 8.5,6.5\rangle+\langle 2.5,1.5\rangle+\langle 5.5,3.5\rangle+\langle 5.5,3.5\rangle
\end{aligned}
$$

The minimum total cost of assigning intervals is achieved by replacing the optimal assignment solution described above in the cost objective interval function as [15.5, 28.5]. It should be noted that our [15.5, 28.5] solution is a lot of more rigorous than the M. Radha, S. Ananthalakshmi, And R. Usha Parameswari [15] solution[19, 30].

\section{CONCLUSIONS}

In this paper we proposed interval versions of Hungarian approach to solve problems with the assignment interval without 
converting them into classical assignment issues. The main aim is to shape the solution more accurately. It is worth noting that the ideal solution obtained through our method is sharper than the solution obtained from others. Numerical cases are resolved and subsequent results are compared to demonstrate the usefulness of the proposed solution.

\section{REFERENCES}

1. A. N. Jasim: A New method to solve Assignment Models, Applied Mathematical Sciences, 11 (54) (2017), 2663-2670.

2. S. Deepa, A. Rameshkumar: New Interval Linear Assignment Problems, IOSR Journal of Business and Management (IOSR$J B M), 20(9)(2018), 5-8$.

3. H. A. Taha: Operations Research (Eighth Edition), Pearson, 2008.

4. Dutta, P. A. L. A. S. H. "Combined approach to propagate aleatory and epistemic uncertainty in risk assessment." Int. J. Math. Comput. Appl. Res 3.5 (2013): 2249-6955.

5. G. RAMESH, K. GANESAN: Assignment problem with Generalized Interval Arithmetic, International Journal of Engineering Research, 6 (3) (2015), 81-85.

6. Amutha, S., Lakshmi, S., Narmatha, S. "Method of Solving Extension Interval in Assignment Problem", International Journal of Engineering Science and Computing, (2017), Vol.7.

7. Cloud, Michael, J., Moore, Raman, E., Kearfott, Baker, E. Introduction to Interval Analysis, Society for Industrial and Applied Mathematics, ISBN-0-89871-669-1, (2009).

8. Anusuya, V., and R. Sathya. "Type reduction on fuzzy shortest path problem." IJMCAR 4.6 (2014): 53-60.

9. Aldous, D. "Asymptotics in the random assignment problem," Probability Theory and Related Fields, 93, (1992), 507-534.

10. Pardalos, P. M. and Pitsoulis, L. (Eds.),Nonlinear Assignment Problems: Algorithms and Applications, Kluwer Academic Publishers. (2000).

11. K. Ganesan and P. Veeramani, On Arithmetic Operations of Interval Num-bers, International Journal of Uncertainty, Fuzziness and Knowledge - Based Systems, 13 (6) (2005) 619 - 631.

12. K. Ganesan, On Some Properties of Interval Matrices, International Journal of Computational and Mathematical Sciences, 1 (2) (2007) 92 - 99.

13. Mraz F., Calculating the exact bounds of optimal values in LP with interval coefficients, Annals of Operations Research, 81 (1998) 51 - 62.

14. D Priya and G Ramesh, The Hungarian Method for the Assignment Problem, With Generalized Interval Arithmetic and Its Applications, Journal of Physics: Conference Series, 2019 J. Phys.: Conf. Ser. 1377012046

15. Gandhi Mallela, Pallavi Paturu, and M. Komaleswarao. "Lift and drag performance of NACA0012 airfoil at various angle of attack using CFD." International journals of mechanical and production engineering research and development 8.3 (2018): 89-100.

16. Atanu Sengupta and Tapan Kumar Pal, Theory and Methodology: On comparing interval numbers, European Journal of Operational Research, 27 (2000), 28 - 43.

17. Ming Ma, Menahem Friedman and Abraham Kandel.,. A new fuzzy arithmetic. Fuzzy sets and systems. 108: 1999, 83-90.[23].

18. Gajivaradhan, P., and S. Parthiban. "Two sample statistical hypothesis test for trapezoidal fuzzy interval data." International Journal of Applied Mathematics \& Statistical Sciences (2015).

19. M. Radha 1, S. Ananthalakshmi, And R. Usha Parameswari, A Modern Approach For Solving Interval Based Assignment Problem, Advances in Mathematics: Scientific Journal 9 (2020), no.5, 2541-2545. 